\title{
Proyecto 2000+: cambiando la enseñanza de la ciencia para el siglo XXI
}

\author{
Jack Holbrook*
}

La Conferencia Mundial sobre Educación para Todos (WCEFA 1990) declaró que

\footnotetext{
“... cada persona... podrá beneficiarse de las oportunidades educacionales diseñadas para satisfacer las necesidades básicas de aprendizaje. Estas necesidades comprenden ambas herramientas esenciales, (tal como el alfabetismo) y los contenidos básicos de aprendizaje (conocimiento, técnicas, valores, y actitudes) requeridas por los seres humanos para participar activamente en el mejoramiento de la calidad de sus vidas, para tomar buenas decisiones y continuar aprendiendo".
}

Esta claro en la anterior cita que los intereses educacionales actuales necesitan estar relacionados con la preparación de los ciudadanos, los cuales son alentados para conducir vidas productivas y disfrutar de la mejor calidad de vida posible. Para conseguir esto, necesitamos resolver problemas sociales que intervienen en nuestra tarea, tales como población, salud, nutrición, medio-ambiente, y un desarrollo sustancial a nivel local, nacional e internacional. Esta tarea social requiere ciudadanos que tengan un grado creciente de alfabetismo científico y tecnológico, ambos para comprender la tarea y la toma de decisión involucra estimular la acción necesaria. Todavía, parece que en muchos países la educación básica en las escuelas incluye muy pocas cosas que ayuden a los estudiantes a

* Ph. D, secretario ejecutivo del Consejo Internacional de Asociaciones para la Enseñanza de la Ciencia (ICASE)

Trad.: Javier Diaz 
adquirir tal alfabetismo, o sentirse seguros de enfrentarse con esta tarea social y la necesidad de tomar responsabilidades para las acciones.

Shymansky y Kyle (1990), en un reporte estableciendo una agenda de investigación para los curricullum de ciencia, lo expresaron de esta manera, "desde que la ciencia y la tecnología están invadiendo más y más nuestras vidas cotidianas, asegurar el alfabetismo en estas áreas en la juventud actual debe ser una prioridad en la reforma educativa"

En un intento para fomentar más pensamientos sobre educación a través de los campos de la ciencia y la tecnología, ambos dentro del sistema formal y para todos los ciudadanos, UNESCO y ICASE iniciaron el Proyecto $2000+$. Su meta es fomentar un mayor nivel de alfabetización científica y tecnológica para todos como una prioridad educacional. Reconoce la necesidad de prestar atención a la educación de todos los ciudadanos y que ya no es apropiado ser guiados por prioridades más relevantes para el pasado. Este trabajo describe el Proyecto 2000+, tareas específicas e investigaciones a ser emprendidas, y considera la manera en la cual la enseñanza de la ciencia necesita cambiar para lograr las metas del Proyecto 2000+ en el siglo XXI.

\section{Proyecto 2000+}

El Proyecto 2000+ se propone fomentar el soporte mundial para la acción en la cual colaboran cuerpos gubernamentales y no gubernamentales, a nivel regional o nacional, para lograr una mayor nivel de alfabetismo científico y tecnológico para todos, el cual es necesario para el siglo XXI. Una Declaración (UNESCO, 1993a), propuesta por participantes en un foro internacional, intentó remarcar la acción necesaria de los gobiernos y otros cuerpos. Más específicamente, el Proyecto 2000+ busca:

a) Identificar formas de fomentar el desarrollo de la alfabetización científica y tecnológica para todos;

b) Proponer programas educacionales (tanto formales como no formales) de manera tal de capacitar a todos para que puedan satisfacer sus necesidades básicas y ser productivos en una sociedad crecientemente tecnológica; 
c) Proveer guías para la continuación del desarrollo profesional de lideres y educadores de ciencia y tecnología;

d) Fomentar la formación de fuerzas nacionales de tareas (involucrando personal de organizaciones gubernamentales e intergubernamentales (OGIs), y de organizaciones no gubernamentales (ONGs) tales como Asociaciones para la Educación científica) para iniciar programas para una mejor alfabetización científica y tecnológica y para identificar y sostener proyectos que fomenten aspectos de la alfabetización científica y tecnológica;

e) Sostener el desarrollo de una larga cadena de proyectos que planeen mejorar la calidad de vida y la productividad en la sociedad y que conducen a la promoción de la solidaridad y cooperación en el logro de la alfabetización científica y tecnológica;

f) Sostener la evaluación de los programas proyectados y los existentes para asegurarse de que la meta de la alfabetización científica y tecnológica esta siendo lograda.

\section{El objetivo}

Para el año 2001 debe haber en cada lugar, en cada país, estructuras y actividades que fomenten la alfabetización científica y tecnológica para todos (Declaración del Proyecto 2000+, sección 8).

"No existe otro segmento en nuestra cultura que retrase la 'sociedad cotidiana' tanto como el proceso de escolarización. La juventud del siglo XIX se sentirá más confortable en nuestras escuelas que en sus hogares, industrias, negocios, o ciudades." (Shymansky y Kyle, 1990, p.5),

\section{Alfabetizacion cientifica y tecnologica}

Como el significado de lograr la alfabetización científica y tecnológica es un área de debate, podría ser útil proponer los siguientes 17 puntos a considerar. Estos puntos fueron sugeridos por la Asociación Na- 
cional de Profesores de Ciencia de América (NSTA 1990-91), intentando ilustrar lo que una persona científica y tecnológicamente alfabeta podría ser capaz de hacer. Estos puntos no son definitivos, como fue señalado por Bybee (1997), STL puede ser lograda en varios niveles. La intención aquí es ilustrar la clase de objetivos a los cuales aspiramos, y al mismo tiempo, permitirle al lector compara esto con lo que actualmente es sugerido en los programas de estudios bajo el título de educación científica (o educación de la biología, educación de la química, educación de la física).

NSTA sugiere que una persona científica y tecnológicamente alfabeta:

1. Usa conceptos de ciencia y tecnología, tanto como conscientes reflexiones de ética incluyendo trabajo y tiempo libre.

2. Se compromete en acciones personales y civiles luego de calcular las posibles consecuencias de las opciones alternativas.

3. Defiende decisiones y acciones usando argumentos racionales basados en evidencia.

4. Toma parte de la ciencia y la tecnología por el entusiasmo y las explicaciones que prevén.

5. Muestra curiosidad y apreciaciones sobre el mundo natural y el hecho por el hombre.

6. Aplica escepticismo, métodos cuidadosos, razonamiento lógico, y creatividad en la investigación del universo observable.

7. Valora la investigación científica y la resolución de problemas tecnológicos.

8. Localiza, colecciona, analiza, y evalúa recursos sobre información científica y tecnológica y usar estos recursos en la resolución de problemas, toma de decisiones, y emprendimiento de acciones.

9. Distingue entre evidencia científica y tecnológica y opinión personal, y entre información fiable y no fiable.

10. Se mantiene abierto a nueva evidencia y al nuevo conocimiento científico y tecnológico experimental.

11. Reconoce que la ciencia y la tecnología son conocimientos humanos.

12. Mide los beneficios y la carga teórica del desarrollo científico y tecnológico.

13. Reconoce la longitud y las limitaciones de la ciencia y la tecnología para avanzar sobre el bienestar humano.

14. Analiza las interacciones entre ciencia, tecnología y sociedad. 
15. Conecta la ciencia y la tecnología con otros comportamientos humanos, por ejemplo historia, matemáticas, las artes y las humanidades.

16. Considera los aspectos políticos, económicos, morales y éticos de la ciencia y la tecnología y como estos se relacionan con los problemas personales y globales.

17. Ofrece explicaciones de los fenómenos naturales cuya validez puede ser testada.

Parecería que existe un gran bache entre las intenciones de los programas de estudio actuales para materias de ciencia y tecnología y las metas de la alfabetización científica y tecnológica expuestas con anterioridad.

Parecería que subdividiendo las materias científicas en divisiones hechas por el hombre tales como biología, química y física, poseen una pequeña ventaja con relación a lo anterior y en efecto existirían muchas más cosas para decir para combinar el futuro programa de estudios científico y tecnológico.

Especialmente donde la educación se relaciona con todos los estudiantes. Ciertamente parecería que la explicación del plan de enseñanza en término de contenidos es inapropiado. Y donde este es el problema, es probable que se llegue a programas de estudios sobrecargados que dificultan el logro de la alfabetización científica y tecnológica para todos pero académicamente es correcto. Finalmente parecería que una variedad de técnicas necesitan ser desarrollas para fomentar el aprendizaje centrado del estudiante, relacionado con las técnicas de pensamiento, investigación y comunicación.

\section{El camino a seguir}

\section{Examinando las tareas}

Mientras la propuesta de la alfabetización científica y tecnológica (llamada anteriormente STL) ha sido establecida y expuesta la indicación de su significado, y mientras más investigaciones en educación científica y la tecnología han brindado sugerencias para el desarrollo de los programas de estudio y la popularización de la ciencia y la tecnología, la dirección actual y el método de operación para lograr las metas del Proyecto 2000+ están claros. 
Los problemas tales como los siguientes son los de mayor importancia para la educación del siglo XXI y necesitan ser discutidos:

a) La naturaleza de la alfabetización científica y tecnológica, cómo lograrla, qué mecanismos de evaluación son más validos;

b) El grado en el que la ciencia y la tecnología deberían ser directamente relevantes a los intereses y demandas de la sociedad y cómo debería ser estudiada como una búsqueda con sus propias estructuras inherentes;

c) La interrelación entre la educación científica y la educación tecnológica; y su lugar como parte integral del programa de estudios escolar formal;

d) La pobre representación, en algunos países, de las mujeres en los cursos científicos y tecnológicos; la escasa representación de mujeres en la práctica científica y tecnológica, en lugares donde son tomadas decisiones sobre ellas; y la exclusión de los beneficios que otorga la ciencia y la tecnología;

e) La manera de popularizar la ciencia y la tecnología en el público general y para eliminar la falta de entendimiento de la ciencia y la tecnología relacionada con los problemas que afectan la sociedad;

f) El énfasis en el curriculum de la educación científica y la tecnología y el impacto de la investigación en el aprendizaje de la ciencia en los niños y las ideas sobre constructivismo;

g) Los métodos de enseñanza y los materiales de enseñanza que luchan por lograr las metas educacionales apropiadas para la alfabetización científica y tecnológica, tanto en los dominios cognitivos y afectivos.

h) El involucramiento de los profesores en la reforma y la aceptación y preparación de los profesores para hacer frente al cambio ante la alfabetización científica y tecnológica para todos;

i) El rol de los libros de texto en la necesidad de relevancia social y con el traspaso de un cuadro de trabajo conceptual fundamental en el cual construir el programa de estudios;

j) Las reformas necesarias de las evaluaciones para asegurar que la ésta se conecte con las metas previstas. 


\section{El rol del ministerio de educación}

El aspecto local del Proyecto 2000+ se propone operar mediante PROGRAMAS NACIONALES a nivel gubernamental, no gubernamental o ambos. Estos programas son planeados localmente, dirigidos locales, implementados localmente, y evaluados localmente. El rol del ministerio de educación es ser consiente de la necesidad de la alfabetización científica y tecnológica (y además reconocer el Proyecto 2000+), y buscar maneras para implementar una mejor educación para todos. Al mismo tiempo, el ministerio de educación necesita fomentar desarrollos no gubernamentales, particularmente instituciones educacionales y asociaciones de profesores de ciencia para iniciar proyectos, proveyendo información, material, y respaldando la operacionalización de la STL en las aulas. Aquellas aulas con un bajo grado de implementación de las intenciones de los programas de estudio (otros conceptos científicos), sabrán que la operacionalización de cualquier filosofía en un aula necesitara toda la ayuda que pueda conseguir de recursos gubernamentales y no gubernamentales.

\section{Los PROGRAMAS NACIONALES podrían:}

1. Adaptar, equipar o reestructurar loa centros existentes o brindar facilidades para sostener el crecimiento de la educación científica y tecnológica a lo largo del país, o crear nuevos centros si es necesario.

2. Sostener el establecimiento o refuerzo de asociaciones profesionales de profesores de ciencia y tecnología, los cuales harán importantes constituciones para lograr tanto las metas cualitativas como las cuantitativas del Proyecto 2000+.

3. Sostener los grupos o instituciones que trabajan y desean unirse a la popularización de la ciencia y la tecnología (museos, exhibiciones, los medios, etc.)particularmente para ayudarlos a focalizar las necesidades de las personas y establecer buenas conexiones con el sistema educacional.

4. Publicitar entre el público en general la idea de una mayor alfabetización científica y tecnológica para todos. 
5. Fomentar el status de la educación científica y tecnológica dentro de la comunidad, usando el sector escolar formal, los medios, y un mayor involucramiento de la comunidad.

6. Emprender un re-pensamiento de la política para la educación científica y tecnológica, remarcando la tendencia a investigar y la creciente importancia de la educación científica como parte integral de las metas generales de la educación.

7. Diseñar nuevos programas de estudios, estrategias impleméntales, materiales de recursos, técnicas de evaluación para sostener el aprendizaje formal, informal y no formal.

8. Fomentar instrumentos de evaluación más validos para usar con los estudiantes y prestar mayor atención a la evaluación de los programas para una conciencia y alfabetización científica y tecnológica.

\section{El rol de las Asociaciones Profesionales de Profesores}

Una Asociación Profesional de Docentes es un grupo de educadores de ciencia unidas en el propósito de fomentar la enseñanza de materias científicas más allá de los aspectos de contenido y de esta manera perfeccionar la educación para los estudiantes. Es voluntario, y las asociaciones pueden aceptar cualquier educador de la ciencia, de los profesores, de los profesores estudiantes, de personas dedicadas al desarrollo del curriculum, de investigadores, etc. Ejemplos notables son; Asociación Nacional de Profesores de Ciencia en USA (NSTA), y la Asociación de la Educación en Ciencia en el Reino Unido (ASE). También existen ejemplos de grupos educacionales profesionales como parte de la sociedad nacional de ciencia (sociedad biológica, sociedad química, sociedad física), a pesar que aquí es más probable poner mayor énfasis en el lado conceptual de la ciencia que en las técnicas profesionales relacionadas en la actual implementación de las intenciones curriculares.

Las mejores formas en las cuales las asociaciones de profesores pueden ser involucradas en la promoción del Proyecto 2000+, y además mejorar la alfabetización científica y tecnológica son:

(a) Convenciendo a los profesores a sostener la nueva dirección y ayudarlos a desarrollar su potencial educacional; 
(b) Desarrollando material y otros recursos para ayudar a los profesores a prepararse para la nueva dirección y alentando a los profesores a probarlos en las aulas;

(c) Proveyendo apoyo durante las clases a través de seminarios o talleres para introducirlos en los planes de prueba y evaluar las estrategias y los materiales relacionados a la nueva dirección;

(d) Proveyendo recursos, los cuales pueden ser encontrados útiles por parte de los profesores en su actualización;

(e) Ejercer presiones en el Ministerio de Educación para emprender acciones conjuntas para fomentar las ideas y calmar los intereses de los profesores;

(f) Jugando un rol de líder sugiriendo, iniciando e implementando proyectos.

Una aproximación importante en la persuasión de los profesores es a través del desarrollo de materiales de enseñanza que son atractivos, fáciles de usar y altamente motivantes. Estos materiales pueden estar directamente relacionados a los programas de estudio por ejemplo la reorientación de las aproximaciones de los profesores que tienen como su propia meta

(i) mayor relevancia en la vida cotidiana

(ii) valores educacionales mayores en la adquisición de las técnicas

(iii) mayor involucramiento por parte de los estudiantes

(iv) una mirada más lógica e interesante sobre los puntos de vista de los estudiantes, o mediante la promoción del profesionalismo de los profesores, los materiales pueden ser desarrollados por profesores para profesores.

El rol de las instituciones de investigación (y Asociaciones Profesionales de Profesores)

Mientras muchas investigaciones en el área de la sociedad científica y tecnológica y en la enseñanza del constructivismo han sugerido adquisiciones positivas, las investigaciones generalmente has sido llevadas a cabo en países desarrollados con estudiantes motivados. Existe la necesidad de conducir investigaciones en ámbitos culturales más amplios.

Las investigaciones son necesarias en áreas tales como:

(a) Las aproximaciones a los programas de estudios necesitan lograr la alfabetización científica y tecnológica así también como técnicas de pensamiento de mayor orden;

(b) Mecanismos de evaluación validos para determinar el logro de niveles de alfabetización científica y tecnológica; 
(c) La extensión de las divisiones hechas por los hombres en materias científicas tales como: biología, química y física son inapropiadas para la alfabetización científica y tecnológica, también, cuan lejos la educación tecnológica debería ser parte integral de los curriculum formales escolares y probablemente pensados separados de la ciencia; Mientras los componentes de la ciencia y la tecnología son únicos, existen también áreas que se superponen y el interés por la alfabetización dentro de estas áreas, en las cuales las tendencias científicas se inclinan sobre la sociedad, están usualmente fuertemente interrelacionadas. Para países desarrollados, la fuerte presión para proveer facilidades especiales (tales como las demandadas por la ciencia pura), la atracción de una tecnología y ciencia mezcladas para todos, podría no ser lo único deseable, pero podría ser doblemente atractiva cuando las consideraciones son dadas a facilidades menos caras, más flexibles.

(d) La enseñanza interdisciplinaria que permite una apreciación y un sentido de responsabilidad por el medio ambiente, por problemas de energía, recursos, salud, alimentos e industrias. Esto conduce a la educación a un desarrollo sustancial y como puede ser logrado, dando esto el mundo se une en intereses tales como, cambio global, deterioro de la capa de ozono, la extinción de las reservas de energía y el impacto de la deforestación;

(e) Problemas asociados con el aprendizaje de la ciencia y la tecnología en los modos y maneras de superar esto. Muchas investigaciones han sido conducidas en esta área y mucho se sabe ahora de las dificultades asociadas con la enseñanza de los conceptos científicos. Sin embargo existen efectos culturales que significan que la investigación en un área no es necesariamente aplicable a otra.

(f) Buscar para proveer una gran cadena de experiencias aplicables tanto a varones como a mujeres. Tradicionalmente la educación científica atrajo a los niños, tanto ofreciéndoles opciones a una edad en la cual los niños son mas fácilmente motivados en dirección a la ciencia. Orientando los temas de la ciencia a estudiar de manera tal que le interese a las niñas, las investigaciones muestran que ellas adquieren igual que los niños. La igualdad en la educación científica significa reconocer la importancia de la educación científica y la tecnología para todos los ciudadanos.

(g) Reestructurar el entrenamiento previo constante de los profesores para hacer frente al cambio. La tarea aquí no es solamente afectar el cambio duradero en el punto de vista y la práctica de los profesores, sino también influir de manera tal que los educadores de profesores (los formadores de formadores) observen la importancia de la alfabetización científico tecnológico y la manera en la cual esta puede ser lograda. Podría también existir una necesidad, como primer paso en el proceso, de educar a los educadores de los profesores. Esto aumenta el problema acerca de: sí los educadores de profesores de las universidades deben ser miembros de un 
departamento de una materia o si deben ser agrupados en una facultad de educación.

El rol de los centros - museos de ciencia y tecnología.

Si nosotros nos comprometemos con la alfabetización científica y tecnológica, cuatro "vehículos" para alcanzarla mediante la educación no formal e informal pueden ser (UNESCO, 1993B):

(a) Actividades científicas tecnológicas fuera de la escuela

(b) Masificar los medios

(c) Instituciones de ciencia y tecnología informal: museos y centros científicos y tecnológicos

(d) Programas basados en la comunidad.

Mientras (a) puede ser dejada como parte del sector de educación formal, mucho se puede decir del valor de los centros científicos tecnológicos (c). Ha ocurrido un cambio dramático de las exhibiciones "no toques" a exhibiciones con actividades interactivas. Algunos centros han olvidado nuevas conexiones con sus regiones y comunidades locales y han establecido las bases de redes regionales de ciencia. Mediante el establecimiento de fuertes conexiones con escuelas, estos centros juegan un rol importante en la conducción hacia la alfabetización científica tecnológica, no sólo proveyendo un estimulo interesante sino interactuando con el trabajo de las escuelas y de esta manera proveyendo recursos adicionales.

\section{Cooperación internacional}

Los gobiernos son miembros de UNESCO y además UNESCO puede actuar como un paraguas para el desarrollo nacional y regional. Puede ofrecerse de guía a través del uso de consultores y la promoción de seminarios nacionales y regionales. Puede diseminar información a través de sus canales de folletos y boletines y puede ayudar a financiar el cambio a través de su participación en programas nacionales y a través de la adquisición de fondos en deposito.

UNESCO, puede conectarse con organizaciones no gubernamentales internacionales. Los siguientes son miembros del comité internacional de 
conducción para el Proyecto 2000+ y están de esta manera deseosos de ayudar a sostener y guiar el desarrollo regional e internacional.

(a) GASAT (Niñas, Ciencia y Tecnología)

(b) ICASE (Consejo Internacional de Asociaciones para la Educación Científica)

(c) ICSU (Consejo Internacional de Ciencias)

(d) WOCATE (Consejo Mundial de Asociaciones para la Educación Tecnológica).

ICASE es una organización mundial paraguas para asociaciones profesionales, institutos, centros, etc. Con un interés en la ciencia, o en la educación científica tecnológica a nivel primario, secundario o a nivel profesores. Se dedica a conectar dichas organizaciones, comentando el intercambio de información e ideas y promocionando el desarrollo para mejorar la educación científica tecnológica.

ICASE no tiene miembros individuales (a pesar que las personas se pueden suscribir a su boletín trimestral). Los miembros existen a través de grupos regionales nacionales o sub-nacionales interesados en la educación científica. Estos grupos pueden ser asociaciones de profesores (miembros totales), instituciones como universidades, centros científicos, museos científicos (miembros institucionales), o compañías de educación científica, fundaciones y organizaciones. Estos grupos integran los miembros de ICASE y eligen un Comité Ejecutivo Honorario para supervisar el funcionamiento de ICASE.

Como un co-iniciador del Proyecto 2000+, ICASE está fuertemente involucrado en la promoción de la alfabetización científica tecnológica y en encontrar maneras de ayudar a los profesores a operacionalizar esto en las aulas. ICASE siente que de esta manera se proveerá a los estudiantes de una educación científica más relevante interesante y efectiva.

\section{Educacion para la alfabetizacion cientifica y tecnologica (STL)}

De acuerdo con ICASE, la educación STL se basa en la preparación de los ciudadanos para la vida dentro de la sociedad tanto para la actualidad como para el futuro. Mediante la preparación para la vida dentro de la 
sociedad, se pretende que los estudiantes adquieran técnicas y actitudes para ser capaces de:

(a) Emprender más elecciones de educación y asegurarse con mayor probabilidad la obtención de empleo;

(b) Funcionar de una manera social positiva dentro de la sociedad;

(c) Adaptarse a, y prepararse para, el cambio en el futuro;

(e) Jugar un rol responsable como ciudadano dentro de la sociedad.

La meta de la educación científica puede ser seguramente nada menos que aspirar alfabetización científica y tecnológica. Esto significa que es mucho más humano, llevando esta aproximación que una búsqueda académica, a pesar de esto dicha meta conduce a la adquisición de conceptos científicos. Una meta importante de la enseñanza de la ciencia es lograr el desafío de operacionalizar la STL.

\section{Los profesores enseñan a los estudiantes, no la ciencia}

Más aún, la aproximación para combatir los tremendos cambios de la tecnología moderna, ha sido diseñar nuevos programas de estudios para incorporar una imagen mas actualizada mediante la inclusión de temas tales como tecnología de la información, control tecnológico, biotecnología, plástica, electrónica, etc.

Pero seguramente esta no es la respuesta. Esto meramente moderniza los contenidos. La educación científica aún lleva una imagen solitaria de las necesidades del ciudadano común. El entendimiento conceptual esta aún en dirección hacia una mirada interna de las disciplinas. Y si nosotros no somos cuidadosos, la ciencia continuará estando asociada con la contaminación, peligros y problemas de salud.

¿Entonces cuál es la respuesta? Obviamente no sería justo decir que una aproximación puede ser la única respuesta. Cambiando la opinión del público, intentando combatir la ignorancia del rol de la educación científica es certeramente el factor. Pero el factor propuesto aquí, el cual no ha recibido la atención que se merece, es probablemente el mayor componente para la falta de cambio en enseñanza de las materias científicas escolares. Ese factor es la visión de la enseñanza de la ciencia por los propios profesores.

Probablemente más que en cualquier otra área de una materia, los profesores de ciencia perciben su tarea como el problema de la enseñanza de la materia (Aikenhead 1997). Ellos ven su tarea como impartiendo el conocimiento y entendimiento de los principios que delinean este cono- 
cimiento (Romberg 1985). El sentimiento es tan fuerte "completar el programa de estudio", como se ejemplifica en los libros de texto, es un llanto común de un profesor de ciencia. Se les da a las investigaciones experimentales una baja prioridad, especialmente si las evaluaciones externas ocupan un lugar de poco peso en esta área. La asistencia a los estudiantes para que desarrollen técnicas de resolución de problemas no es tomada en serio, a menos que esto simplemente signifique taclear una variedad de problemas numéricos. Certeramente el tiempo no debe estar a un lado para planear una investigación y luego llevarla a cabo o para una situación de toma de decisión donde la respuesta no es clara y una multitud de factores pueden influir en dicha decisión, tales como factores económicos, sociales, medio ambientales, políticos, o consideraciones éticas.

Parecería que los profesores continúan enseñando los contenidos de la materia por una variedad de razones. Un factor importante es que los libros de texto, para la mayor parte, ignoren áreas no conceptuales sólo incluyendo aplicaciones dentro de la sociedad de los conceptos científicos estudiados, en vez de empezar por la forma en que la sociedad puede utilizar la ciencia. El énfasis y aproximación de libros de texto está fuertemente influenciado por la manera en que los programas son diseñados por conveniencia, los programas de estudios están casi invariablemente diseñados sobre la base de fundamentos científicos, agrupando conceptos científicos. Los programas de estudios raramente agrupan el aprendizaje basándose en la manera en que la sociedad puede hacer uso de la ciencia, por ejemplo para solucionar inconvenientes - problemas en la cocina, el baño, el medio ambiente local, industria y empleo.

\section{Evitando el cambio}

La evidencia sugiere que los profesores tratan de evitar el cambio, especialmente aquel que significa que su experimentación podría estar socavada (Aikanhead, 1997). Los profesores de ciencia no han tenido que tratar, en el pasado, con el desarrollo de técnicas de solución de problemas o tomas de decisiones útiles para la sociedad. Los profesores, en el pasado, no han tenido que tratar con el desarrollo de los estudiantes como miembros de la sociedad, o con el desarrollo de técnicas sociales (para ser un miembro responsable de la sociedad) durante las lecciones de ciencia. De esta manera a los profesores de ciencia no les es fácil introducir valores éticos o sociales en las aulas científicas, aún cuando esto tiene gran relevancia para la sociedad (Gaskell, 1982). 

cambio?

¿Entonces qué sucede si se contempla un curriculum conducente al

Si el curriculum intenta introducir una gran cantidad de técnicas educacionales y por esto, reduce los contenidos científicos, los profesores tienden a utilizar este tiempo extra para desarrollar más profundamente en el problema de la materia. Los profesores evitan la incorporación de actividades para los estudiantes centradas en lluvias de ideas, juegos de roles, pequeños grupos de discusión y actividades creativas, tales como el desarrollo de pósters, composición de cartas a organismos oficiales, acrecentando la conciencia medio ambiental y la participación en debates. Esto no mueve los contenidos hacia delante. El conocimiento práctico de los profesores determina la práctica en el aula (Duffee y Aaikenhead, 1992). Todavía son evitadas las actividades que tienen el potencial para acrecentar el contenido de conocimientos, por ejemplo búsqueda en biblioteca, ubicación de orígenes secundarios de información, porque esto no está bajo el control de los profesores.

Los profesores de ciencia están de esta manera contentos enseñando su materia. Sus esfuerzos son cada vez más irrelevantes para la sociedad moderna. Ellos ignoran la protección de las lecciones de ciencia para una relevancia social mayor. Se les ha entregado un programa de la materia cubriendo cual es su percepción de la tarea. ¡Los profesores enseñan de la manera en que han sido enseñados! (Gregorio, 1997). Como una consecuencia la ciencia se torna impersonal, cada vez más irrelevante y en las manos de profesores desinteresados, un ejercicio de memoria. Es en el cambio de este punto de vista que las asociaciones de profesores de ciencia necesitan avanzar (Haggis, 1991). Estas áreas olvidadas en la educación científica son importantes para la educación del estudiante. Nada dice que en el pasado los estudiantes encontraban la ciencia difícil, ni que las niñas no tendieran a elegir esta opción, ni que los profesores de escuelas primarias se escondan de enseñar ciencia.

¿ Entonces que podemos hacer? ¿ Cómo operacionalizar la STL?

Ninguna innovación en la educación científica ha sido exitosa sin el soporte y experimentación de los profesores en las aulas (Holbrook, 1996; Pak, 1997; Purkey y Smith, 1982,Rroy Singh, 1991; UNESCO, 1997). Ayudar y sostener a los profesores es crucial.

Primero y principal, está la necesidad de cambiar la percepción de los profesores sobre lo que se trata la enseñanza de la ciencia. Los profesores de ciencia necesitan ser guiados para enseñar a los estudiantes. Y esto significa realizar su parte en el logro de las metas educacionales propuestas para la escolarización. Los profesores científicos deben darse 
cuenta que la educación no es sólo sobre conceptos y contenidos, sino que requiere atención a las técnicas personales y sociales, consideraciones éticas y sobre todo, requiere atención al dominio efectivo. La educación y la ciencia deben ser vistas como parte de la educación.

Mientras esto es fácil de decir no es tan fácil proponer guías sobre cómo puede ser logrado el cambio. Pero al menos el 50\% de la batalla es hacer que los profesores de ciencia perciban la necesidad de cambiar. El curriculum desea que ellos cambien y la sociedad como la parte más importante (la cual influye a través dell Ministerio de Educación) quieren que cambien.

La tarea ante nosotros es lograr que los profesores de ciencia cambien por una nueva imagen de la educación científica -STL para todos-. Esta imagen se ocupa de los estudiantes como individuos. Permite a los profesores desarrollar nuevas estrategias que abarquen un grado mayor de enseñanza centrada en los estudiantes (Gunstone, 1997); Presta más atención a la relevancia en la sociedad, valora la educación y sobre todo, permite a los profesores de ciencia prestar atención al desarrollo de cualidades personales y actitudes positivas.

Sin duda, contemplando el cambio significa que los profesores necesitan soporte continuo. Pero esta progresión continua necesita consideraciones cuidadosas que indiquen las necesidades reales de los profesores (Cho, 1997). Diciéndoles a los profesores que cambien no es probable que ayude a esta situación. Ellos necesitan que se les muestre cómo, y decirles por qué; ésta es la manera en la cual cambiar. Los formadores de los profesores deben convencerse de la necesidad de cambiar y para que el cambio ocurra, los profesores necesitan ser convencidos acerca de cómo el cambio puede ser exitosamente implementado.

\section{Convenciendo a los profesores que se unan al cambio}

\section{Dos factores de especial significado a considerar:}

(a) Un aspecto es proveer evidencia de investigaciones mostrando que el cambio puede llevar a adquisiciones sustanciales para los estudiantes y, que los estudiantes no sufran en su habilidad de seguir cursos de ciencia a un nivel más alto (Boyer, 1983; Byrne y Johnstone, 1988; Goodlad, 1984). 
(Tal evidencia es también necesaria para convencer al personal de las universidades en las facultades de ciencias, que son usualmente los más escépticos de los que están involucrados en el desarrollo de curriculum de ciencias escolares. Los profesores universitarios están preocupados porque los estudiantes no obtendrán el conocimiento para manejarse bien con las demandas de los cursos universitarios de ciencia. Y como consecuencia no desearán continuar, a pesar de esto sólo un muy pequeño número de estudiantes nunca más desearán continuar en la búsqueda de investigaciones científicas) La evidencia de investigaciones actuales, muestran que la búsqueda de técnicas de pensamiento de mayor orden dan al estudiante una mayor recompensa que los ejercicios de memoria, a pesar de que éstos cubren menor material fáctico. La evidencia muestra que las actitudes positivas son enriquecidas por la mayor relevancia social y porque el estudiante está más involucrado con la tarea. También muestran que las actitudes positivas conducen a logros académicos mayores. Y con exámenes bien preparados o los indicadores de evaluación de los estudiantes adquieren mas cuando muestran una actitud mas positiva hacia la materia y son capaces de usar una cantidad de técnicas educacionales (Kellerman, 1993; Liu, 1997; McCormack, 1996; Weiss, 1993).

(b) Un segundo factor es proveer cursos continuos apropiados (Yager, Hidaayat and Penick, 1988). Una aproximación que muestra signos de éxitos es hacer que los profesores reflexionen sobre el cambio necesario en la educación científica y luego con esto en mente, desarrollar materiales suplementarios de enseñanza. Los talleres para profesores que prestan gran atención STL, han sido desarrollados por ICASE (Holbrrok y Rannikmae, 1997) y los materiales derivados de estos talleres están empezando a aparecer en Internet (UNESCO Resourse kit, como una fase piloto en la siguiente dirección: http: //www. canterbuy.ac.uk / depts /acad/teacher/ICASE/icase.htm). Dándoles a los profesores la autoría de esta filosofía mediante la creación de los profesores de materiales suplementarios de enseñanza que se unen al criterio STL, este es el foco de los talleres, a pesar que la meta principal es dar a los profesores autoría de esta filosofía que se expande. 


\section{EI criterio para los materiales ejemplares STL}

\section{El criterio sugerido para la creación de materiales suplementarios de enseñanza ejemplares ( Holbrook, 1997; Holbrook y Rannikmae, 1997) es:}

(a) FOMENTAR EL LOGRO DE UNA CANTIDAD DE METAS EDUCACIONALES POR PARTE DE LOS ESTUDIANTES

Una gran cantidad de metas educacionales son estipuladas y forman el foco principal del material, por ejemplo, los estudiantes participando del proceso de aprendizaje educacional, apropiado para las metas del país y el desarrollo intelectual. Estas metas no sólo se relacionan con conceptos científicos y métodos científicos, sino también incluyen valores sociales y el desarrollo de técnicas personales.

(b) COMENZAR DE UN INTERES O PROBLEMA SOCIAL

El material está socialmente relacionado y generado desde un interés o problema dentro de la sociedad. Los estudiantes están familiarizados con la situación, y pueden de esta manara apreciar su relevancia y son capaces de construir sobre constructos ya formados. Los materiales son directamente relevantes a los ojos de los estudiantes. No es sugerido crear materiales sobre presión, o fotosíntesis, porque su relevancia no es pública. Creando materiales sobre la correcta presión en los neumáticos para mayor seguridad, la necesidad de humectación de la piel para una vida saludable, o el problema de la selva Amazónica tropical para precaución global, se estaría siendo relevante a la sociedad. La relevancia estaría clara a pesar que los conceptos científicos pueden no haber cambiado.

(c) INVOLUCRA DEMANDAR TECNIOCAS DE PENSAMIENTO DE MAYOR ORDEN

Emprender la actividad es un ejercicio apropiado de aprendizaje para el aprendiz. Por ejemplo, Provee un desafío intelectual en un nivel apropiado para el estudiante. Utiliza conceptos constructivistas - moviéndose de la información y el entendimiento ya en poder del estudiante, a una nueva situación de aprendizaje. Involucra un pensamiento analítico y evaluativo.

(d) LA PARTICIPACION DE LOS ESTUDIANTES.

La aproximación de enseñanza y las actividades son diseñadas para la participación de los estudiantes. Por ejemplo, el material involucra a los 
estudiantes, tanto individualmente como grupalmente, por un tiempo considerable del tiempo de enseñanza (60\%). Esto no significa necesariamente que los materiales de enseñanza estén orientados (a pesar de que esto sea importante), pero si significa que se presta atención a la incorporación de una variedad de aproximaciones de enseñanza, a través de las cuales los estudiantes pueden participar.

(e) INCLUYE UN COMPONENTE DE TECNICA DE COMUNICACI'N.

Esta consideración se da para mejorar la gran cadena de técnicas de comunicación apropiadas para la diseminación de las ideas y los valores sociales. Esto involucrara formas gráficas, tabulares, simbólicas, pictóricas, así también como escritas (grupos de discusión, debate, juego de roles).

(f) SE RELACIONA CON LA EDUCACION DE LA CIENCIA.

Mientras que los materiales conectan la enseñanza de ciencia con la ciencia social, particularmente por el escenario en el cual el material es desarrollado, la enseñanza aun retiene la adquisición de conceptos científicos y la atención al desarrollo del proceso científico. El material es definitivamente educación a través de la ciencia, no una ciencia social sumada a un componente académico científico enseñado previamente. El desarrollo de las técnicas de procesamiento tales como experimentación son apropiadamente enfatizadas.

\section{(g) INCLUYE UNA GUIA COMPRENSIVA PARA LOS PRO- FESORES}

Como problemas, intereses provenientes de la sociedad son usualmente interdisciplinarios en carácter, con las ideas científicas no familiares para el profesor, explicaciones completas son necesarias para ayudar a los profesores a que usen los materiales de una manera significativa e interesante.

La guía de los profesores también necesita remarcar las conexiones entre actividades propuestas por los estudiantes y los resultados esperados en términos de objetivos educacionales.

La guía de los estudiantes necesita detallar una estrategia sugerido de enseñanza mediante la cual se ejemplifica la aproximación centrada del estudiante.

¿Cómo los profesores deberían adquirir esta dirección y utilizar los materiales STL?

La respuesta sencilla es la participación de los profesores en los cursos continuos que han sido introducidos en el criterio y luego tratar de 
crear sus propios materiales de enseñanza. Si los profesores pueden hacer esto (y no es de ninguna manera una tarea fácil) y los materiales creados últimamente reflejan la nueva dirección de la enseñanza, luego utilizando esto estarían aplicando la STL en las aulas (Rannikmae, 1997). El nuevo material aceptará automáticamente la visión amplia de la educación científica.

Si los profesores eligen no crear el material ellos mismos, pero sí usar materiales existentes, ellos aún necesitan asegurarse de que son conservados con la nueva visión de la educación STL. Esto significa que ellos necesitan aceptar la idea que la educación científica comienza de un interés o problema social antes de progresar con el método científico y los conceptos científicos. Y que la educación científica involucra valores sociales y desarrollo personal, tanto como ideas conceptuales científicas.

\section{RESUMEN}

En un intento para fomentar más pensamientos sobre educación a través de los campos de la ciencia y la tecnología, ambos dentro del sistema formal y para todos los ciudadanos, UNESCO y ICASE iniciaron el Proyecto 2000+. Su meta es fomentar un mayor nivel de alfabetización científica y tecnológica para todos como una prioridad educacional. Reconoce la necesidad de prestar atención a la educación de todos los ciudadanos y que ya no es apropiado ser guiados por prioridades más relevantes para el pasado. Este trabajo describe el Proyecto 2000+, tareas específicas e investigaciones a ser emprendidas, y considera la manera en la cual la enseñanza de la ciencia necesita cambiar para lograr las metas del Proyecto 2000+ en el siglo XXI.

\section{REFERENCIAS}

AIKENHEAD, G. S. Changes Need to Succeed Where We Previously Failed. In: KEDI, Globalization of Science Education - moving toward worldwide Science Education Standards. Proceedings of the International Conference on Science Education, May 26-30, 1997, Korea, 1997.

BOYER, E. High School: A Report On Secondary Education in America. New York: Harper and Row, 1983 
BYBEE, R. W. Achieving Scientific Literacy: From Purposes to Practice. Portsmouth NH: Heinemann, 1997.

BYRNE, M.S. and Johnstone, A.H. How to Make Science Relevant. School Science Review, v. 70, n. 251, p. 43-46, 1988.

CHO, J.-Il. Resources and Their Uses for Science Teacher In-service Education and Science Teaching: Cases of Iowa. In: KEDI. Globalization of Science Education - moving toward Worldwide Science Education Standards. Proceedings of the International Conference on Science Education, May 26-30, 1997, Korea, 1997.

DUFFEE, L. and AIKENHEAD, G. Curriculum Change, Student Evaluation and Teacher Practical Knowledge. Science Education, v.76, n. 5, p. 493-506, 1992.

GASKELL, P. J. Science Education for Citizens: Perspective and Issues 1: Science, Technology Society: Issues for Science Teachers. Studies in Science Education. v. 9 , p.33-46, 1982.

GOODLAD, J. A Place Called School: Prospects for the Future. New York: McGraw-Hill, 1984.

GREGORIO, L.C. Supporting Change of Science and Technology Teacher Perception in the Asian Region. In: KEDI: Globalization of Science Education moving toward Worldwide Science Education Standards. Proceedings of the International Conference on Science Education, May 26-30, 1997, Korea.

HAGGIS, S.(ed.). Education for all: Purpose and Context. Paris: UNESCO, 1991.

HOLLBROOK, Jack.Resource Materials for Science Education Reform. In: KEDI. Globalization of Science Education - moving toward Worldwide Science Education Standards. Proceedings of the International Conference on Science Education, May, 26-30, 1997, Korea.

HOLLBROOK, Jack. The role of Science Teacher Associations in Promoting Scientific and Technological Literacy. Science Education International. v. 7, n. 1, March 1996, p 5-10, 1996.

. Scientific and Technological Literacy for All - The Role of Educators. Science Education International, v. 5, n. 3 , sep. 1994, p. 10-16, 1994.

HOLLBROOK, Jack and RANNIKMAE, Miia (eds). Supplementary Teaching Materials - Promoting Scientific and Technological Literacy. Tartu, Estonia: ICASE, 1997.

ICASE /UNESCO, Project 2000+ - document, 1992.

KELLERMAN, L. R.An Issue as an Organiser: A Case study. In: YAGER, R. E. (ed). The Science, Technology, Society Movement. What Research Says to the Science Teacher, v.7 .Washington D.C.:National Science Teachers Association, 1993.

LIU, Chin-Tang. The Impact of Iowa Chatauqua and Scope, Sequence and Coordination Programs on Teachers'and Students'Changes: A Ten-year Review, 1997.

KEDI. Globalization of Science Education - moving toward Worldwide Science Education Standards. Proceedings of the International Conference on Science Education, May, 26-30, Korea, 1997. 
McCOMAS, W.F. The Affective domain and STS Education. In R.E.Yager (ed). Science/Technology/Society as Reform in Science Education. Albany, New York: SUNY Press, 1996.

NATIONAL Science Teachers Association. Science/Technology/Society: A new effort for providing appropriate science for all (Position Statement). In: NSTA Handbook, p. 47-8, 1990-91.

PAK, Sung-Jae. A Challenging conception of Science Teaching. In: KEDI. Globalization of Science Education - moving toward Worldwide Science Education Standards. Proceedings of the International Conference on Science Education, May, 26-30, 1997, Korea, 1997.

PURKEY, S. C. and SMITH, M. S. Too soon to cheer ? Research on Effective School sand Educational Leadership. Elementary School Journal, n. 40, p. 6469, 1982.

RANNIKMAE, M. Supplementary STS Teaching Materials as Tools for Assessment. In: KEDI. Globalization of Science Education - moving toward Worldwide Science Education Standards. Proceedings of the International Conference on Science Education, May, 26-30, 1997, Korea.

ROMBERG, T. A. (ed) Towards Effective Schooling: The IGE Experience. Lanham, MD: University Press of America, 1985.

ROY SINGH, R.Education for the twenty-first century. Bangkok: UNESCO PROAP, 1991.

SHYMANSKY JAMES A. and KYLE WILLIAM C. Jr. Establishing a Research Agenda: The critical issues of science curriculum reform. Report of a conference, April 1990, p. 2.

UNESCO. Scientific and Technological Literacy within Formal Schooling. Unpublished monograph. Internet address for draft version: http://www.canterbury.ac.uy/depts/acad/teached/ICASE/icase.htm, 1997.

. Resource Kit. Unpublished manuscript. Internet address for draft version: http://www.canterbury.ac.uy/depts/acad/teached/ICASE/icase.htm, 1997.

. Asian STL Teaching materials. Unpublished manuscript, 1998.

. Declaration by participants at an International forum on Scientific and Technological Literacy for all, UNESCO, Paris, 1993 a.

.. Final Report: International forum on Scientific and Technological Literacy, UNESCO, Paris, 1993 b.

WCEFA 1990. Final Report. World Conference on Education for All: Meeting basic learning needs, p. 119.

WEISS, I. R. 1993. Science Teachers Rely on the textbook. In: YAGER, R. E. (ed). The Science, Technology, Society Movement. What Research Says to the Science Teacher, v. 7, Washington D.C. : National Science Teachers Association.

YAGER, R. E.; HIDDAYAT, E. M. and PENICK, J. Features which separate least effective from most effective science teachers. Journal of Research in Science Teaching, v. 25, n. 3, p. 165-167, 1988. 